\title{
Cultivation of Two Species of Mushroom Found in Anambra State, South-Eastern, Nigeria and Their Potentials for Bioremediation of Trace Metals Polluted Soil
}

\author{
Dr. (Mrs) B. C. Ilechukwu'1, Prof. C. O. B. Okoye ${ }^{2}$ \\ ${ }^{1}$ Senior Lecturer, ${ }^{2}$ Professor \\ 1,2Department of Pure and Industrial Chemistry, Faculty of Physical Sciences, \\ 1,2University of Nigeria, Nsukka, Enugu, Nigeria
}

\begin{abstract}
How to cite this paper Dr. (Mrs) B. C. Ilechukwu | Prof. C. O. B. Okoye "Cultivation of Two Species of Mushroom Found in Anambra State, South-Eastern, Nigeria and Their Potentials for Bioremediation of Trace Metals Polluted Soil" Published in International Journal of Trend in Scientific Research and Development (ijtsrd), ISSN: 24566470, Volume-3 | Issue-3, April 2019, pp.1761-1772, URL: https://www.ijtsrd.c om/papers/ijtsrd22 846.pdf

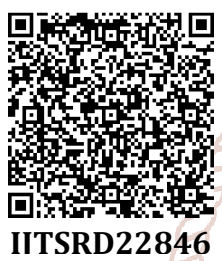

Copyright (C) 2019 by author(s) and International Journal of Trend in Scientific Research and Development Journal. This is an Open Access article distributed under the terms of the Creative Commons

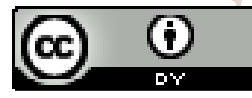
Attribution License (CC BY 4.0) (http://creativecommons.org/license s/by/4.0)

\section{ABSTRACT}

Two species of Mushroom namely: Amanita phalaoides and Amanita verosa were studied and they were collected from farmlands in Uke town around Onitsha metropolis in Anambra State, Nigeria. The mushroom samples were cultivated on normal agricultural and refuse dump soils. The young growing mushrooms were watered twice daily and harvested within fourteen (14) days. The harvested mushroom samples were sun-dried and kept in pre-cleaned bottles for chemical analysis. The dried samples were cut into pieces and pulverized using binatone blender with stainless blade and they were subjected to chemical analysis using standard analytical methods ((AOAC). The obtained data were analyzed by ANOVA using SPSS versions 16.0. Duncan's Range was applied in separating means where differences were observed. The results ranged as follows: moisture content (MC) $81.79 \%$ to $97.84 \%$, the highest value was from Amanita Phalaoides. Dry matter (DM) ranged from $2.63 \%$ to $18.36 \%$ shows an indication of high roughages contained by mushrooms. Crude protein (CP) ranged from $8.16 \%$ to $24.67 \%$ which compared favourably with values of seeds and legumes. Ash contents ranged from $3.26 \%$ to $14.33 \%$ and they are indications of high mineral elements present in mushroom species. Low values of Lipid (fat/oil) ranged from $1.00 \%$ to $6.68 \%$ are indications that mushrooms are excellent dietary food for diabetic and coronary heart disease patients. Crude fibre (CF) ranged from $2.62 \%$ to $15.37 \%$. Values of Ethanol soluble sugar (ESS), carbohydrate ( $\mathrm{CHO}$ ) and vitamin $\mathrm{C}$ were close showing no significant difference at $p>0.05$. Values of phytic acid, tannins and hydrocyanic acid ranged from 0.01 to $0.27 \mathrm{mg} / 100 \mathrm{~g}$, these were quite low to give adverse effect. The mean concentrations of nutrients metals $(\mathrm{mg} / \mathrm{kg}$ ) of $\mathrm{Na}, \mathrm{K}, \mathrm{Ca}, \mathrm{Mg}$ and $\mathrm{Fe}$ ) ranged from $276.48 \pm 0.27$ to $826.83 \pm 0.04$ while trace metals (Cu, Co, Pb, Zn, Cd, Ni, Mn, Cr) concentrations $(\mathrm{mg} / \mathrm{kg})$ ranged from $0.001 \pm 0.01$ to $43.18 \pm 0.20$. Cobalt was most bio-accumulated in agric soil while $\mathrm{Zn}$ was most bio-accumulated in refuse dump soil.

KEYWORDS: Indigenous mushrooms, Amanita phalaoides, Amanita verosa, Anambra State, South-Eastern, Nigeria.

\section{INTRODUCTION}

Mushrooms are a special group of fungi which are saprophytic in nature due to lack of chlorophyll ${ }^{1}$. They grow in dark, damp places and produce a wide range of enzymes which progressively breakdown complex substances into simpler inorganic matter ${ }^{2,3}$. In many parts of the world, such as China, United State of America (USA), Canada, India, Italy, Mexico and Turkey, mushrooms are highly priced and in massive production for local consumption and export ${ }^{6,14}$. In U.S.A, the gross domestic product (GDP) for mushroom was about seven million tonnes in 2005, amounting to 30 million dollars annually ${ }^{16}$. In Nigeria, mushrooms are grossly under exploited as only a few types are considered edible. There is no evidence of mushroom cultivation as a commercial venture in Nigeria, but this could be used as a means of poverty alleviation due to its short cropping cycle, cheap planting inputs, less land requirement, high profit and quick returns on investment ${ }^{17}$. 
Uses and economic importance of mushrooms are illustrated in Fig 2.1.

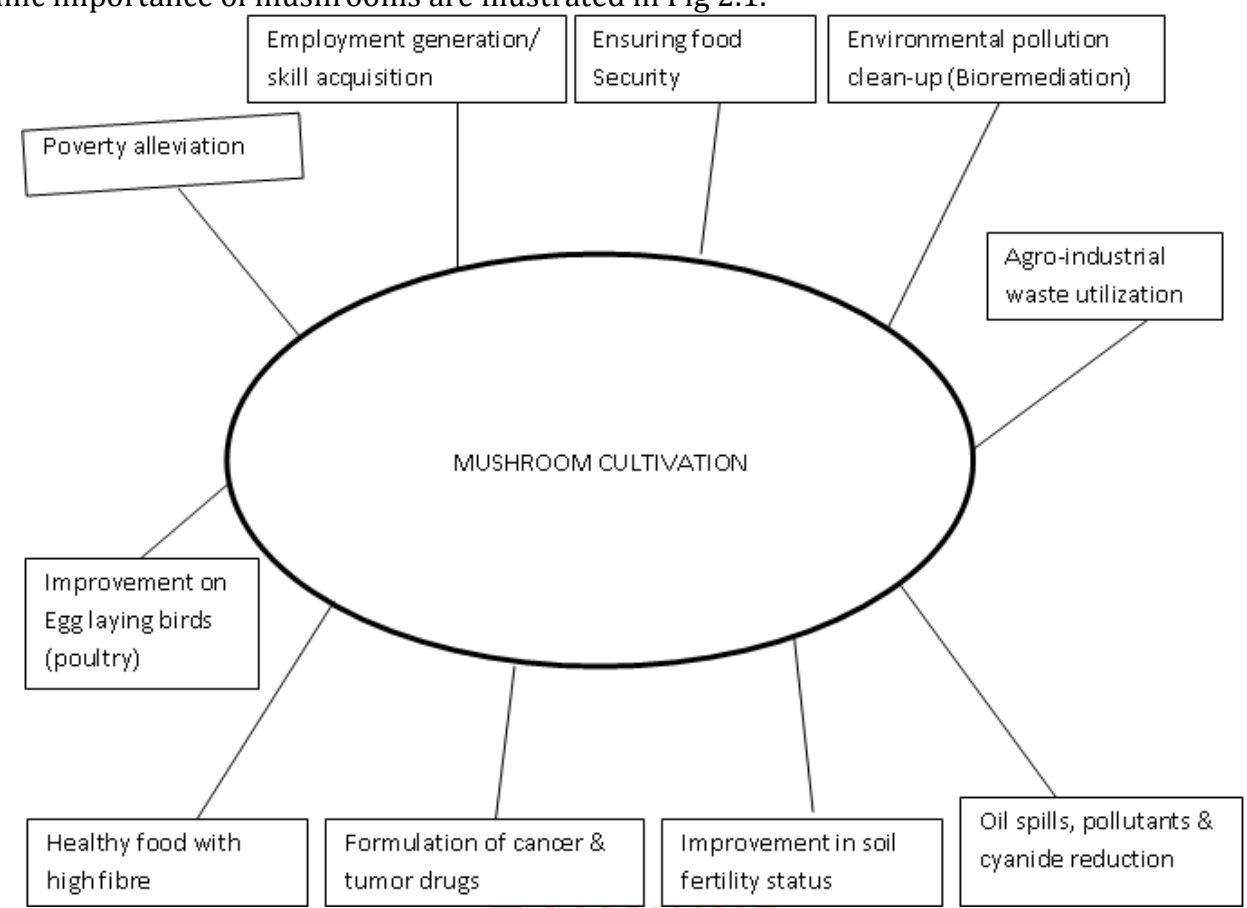

Fig 2.1

Till date, mushroom collection in Nigeria is mainly from the wild and this practice is fraught with fear of mistaking those regarded as poisonous and non-edible for those regarded as edible. This could be occasionally attributed to deaths after mushroom meals. Industrialization, urbanization and indiscriminate refuse disposal have impacted negatively on the environment, thereby posing problems of contamination with pesticides, petroleum hydrocarbons, heavy metals and other potential pollutants ${ }^{21}$. Mushrooms have been reported to be good accumulator of trace metals in polluted environment ${ }^{24}$.

Most Nigerians in the rural areas (65\% of population) eat mushrooms. A close look at the traditional farming system reveals that there has been a place for mushroom production in the country. Traditionally, there are different ways by which our people have consistently maintained an interest in mushrooms. In some parts of the country, the local population do the following to produce mushrooms. This involves taking proper note of the site of occurrence of a mushroom in the growing season (mostly rainy season) such sites are regularly visited for mushroom harvest. The area is protected either by fencing or ring weeding such that it is isolated from other parts of the farm or the forest. It has been estimated that about 140,000 mushrooms are on earth and only $10 \%$ are known. A large number of the unknown mushroom resides in Africa and may be largely in Nigeria because there are no sufficient data on indigenous mushroom in this part of the globe. Mushroom collection from the wild had been in the past and still is a source of income for rural dwellers in Nigerian communities. Therefore, mushroom production as a commercial venture will be an effective means for poverty alleviation. This is due essentially, to its short cropping cycle, low cost production, high profit and quick return. These features of mushrooms could be harnessed in several ways in Nigeria as is being done in other countries such as India, Kenya, Uganda and Zimbabwe for economic empowerment of our people. Mushroom cultivation is a big-time multimillion dollar business abroad. About two decades ago, the world production of cultivated edible mushroom was estimated to be about 7 million tonnes ${ }^{26,27}$. The combined total market value for medicinal and edible mushroom for the same period was estimated to be in excess of U.S. $\$ 30$ billion. In China for example, the production of mushrooms increased steadily over time (Table1). It is being anticipated that in the nearest future, Nigeria will be able to have similar data for comparison.

Table1: Production of Cultivated Mushrooms in China 6-64.70 during the period 1978-2000.

$\begin{array}{lll}\text { Year } & \text { Production(x 1000MT) } & \text { Increase (\%) } \\ 1978 & 60 & - \\ 1986 & 585 & 875.0 \\ 1994 & 2640 & 351.3 \\ 1997 & 3918 & 48.4 \\ 2000 & 6630 & 69.2\end{array}$

(Source: Stamets, $\mathrm{P}(2005)^{13}$

In Africa, it has been reported that even if the production cost for mushroom is doubled it would still remain more profitable than that of maize, wheat, soybean etc. However, the production of mushrooms could be integrated into the already existing agro-system such as maize, wheat and soybean programmes ${ }^{28}$.

In this research work, two unpopular non-edible mushrooms were cultivated on normal agricultural and refuse dump soils. Their nutritional, anti-nutritional and mineral compositions were determined. The aims were to: (i) ascertain their toxicological profiles and edibility (ii) create awareness for greater exploitation of the mushroom species, (iii) to investigate possible use in biotechnology for bioremediation of trace metals polluted soil, and (iv) access their edibility

\section{EXPERIMENTAL METHODS}

Two species of mushroom namely - Amanita phalaiodes and amanita verosa were identified by a taxonomist and collected from farmlands, bush and forest in the vicinity of 
Uke around Onitsha area in Anambra State, South- Eastern, Nigeria. They were kept in clean collection bags, and taken to the laboratory for cultivation. Seed from matured mushrooms were scrapped into two soil samples -normal agricultural (natural habitat) and refuse dump soil. The fruiting bodies were watered twice daily and were harvested within fourteen days. They were thoroughly cleaned, cut into pieces and oven dried at $75^{\circ} \mathrm{C}$ for $6 \mathrm{~h}$. Dried samples were homogenized using binnatone blender with stainless blade into fine powder and stored in pre-cleaned bottles. These samples were subjected to various standard analytical methods to determine their physicochemical and toxicological profiles as recommended by Association of Official Analytical Chemists (AOAC), moisture content and dry matter were determined by oven method, crude protein (kjeldahl apparatus), lipids (soxhlet apparatus), crude fibre and ash (muffle furnace), ethanol soluble sugars (using digital refractometer), carbohydrates by difference and vit. C (titrimetry), cyanide, phytic acid and tannins (UV/visible spectrophotometer), essential mineral elements, and some heavy metals were determined using flame photometer and atomic absorption spectrophotometer (AAS). The obtained data were subjected to analysis of variance at $95 \%$ confidence level using SPSS version16.0.

\section{RESULTS AND DISCUSSION}

The moisture content (MC) ranged from $81.79 \%$ to $97.84 \%$, the highest value was from Amanita Phalaoides. Dry matter (DM) ranged from $2.63 \%$ to $18.36 \%$ shows an indication of high roughages contained by mushrooms. Crude protein (CP) ranged from $8.16 \%$ to $24.67 \%$ which compared favourably with values of seeds and legumes. Ash contents ranged from $3.26 \%$ to $14.33 \%$ and they are indications of high mineral elements present in mushroom species. Low values of Lipid (fat/oil) ranged from $1.00 \%$ to $6.68 \%$ are indications that mushrooms are excellent dietary food for diabetic and coronary heart disease patients. Crude fibre (CF) ranged from $2.62 \%$ to $15.37 \%$. Values of Ethanol soluble sugar (ESS), carbohydrate ( $\mathrm{CHO}$ ) and vitamin $\mathrm{C}$ were close showing no significant difference at $p>0.05$. Values of phytic acid, tannins and hydrocyanic acid ranged from 0.01 to $0.27 \mathrm{mg} / 100 \mathrm{~g}$, these were quite low to give adverse effect..

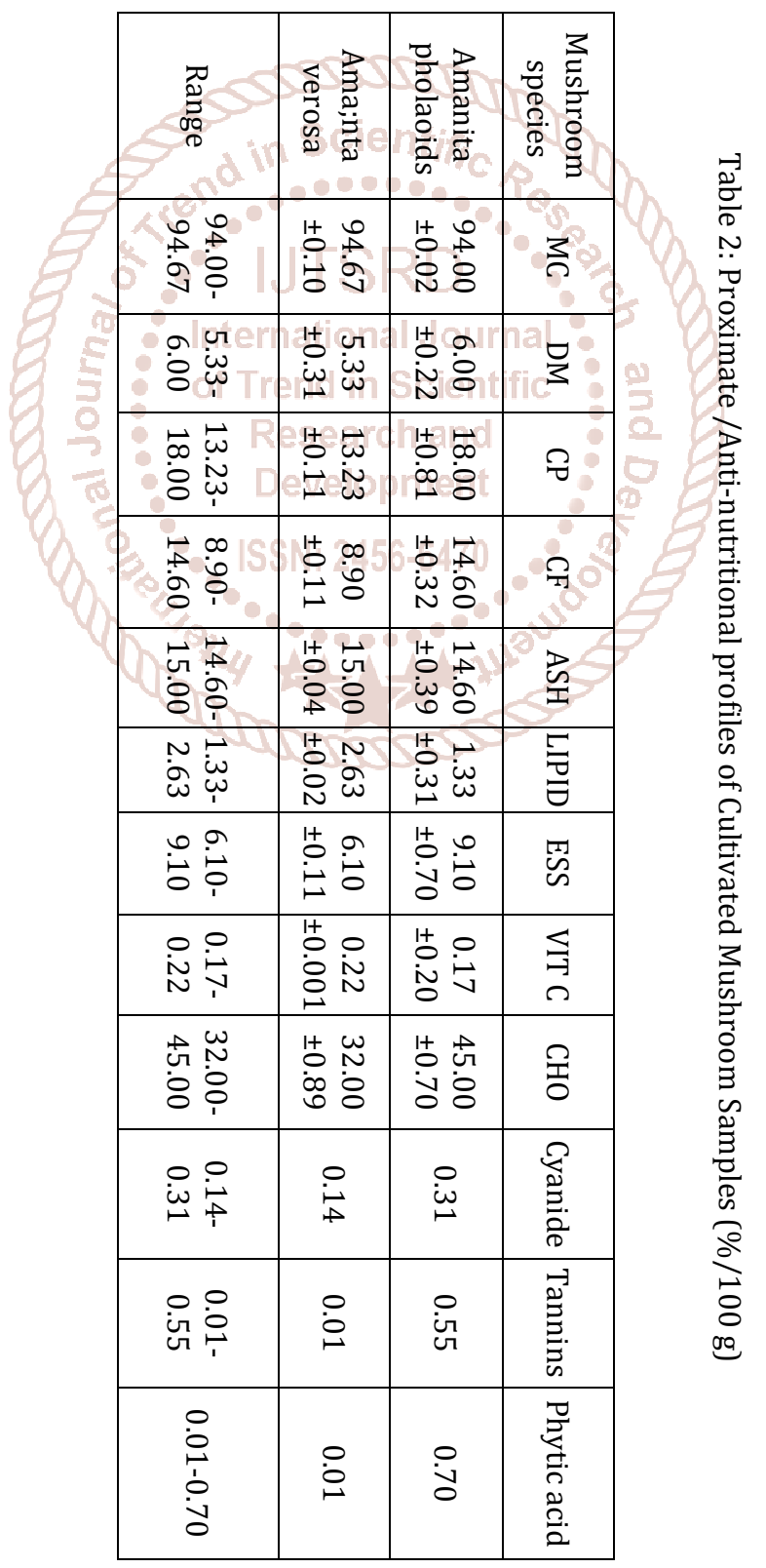

These values were low to give adverse effect and compared well with $1.00 \mathrm{mg} / 100 \mathrm{~g}$ found in (WHO 1995) ${ }^{44}$ guideline levels for vegetables. Table 3 contains the concentrations of essential metals in cultivated mushroom samples. 
International Journal of Trend in Scientific Research and Development (IJTSRD) @ www.ijtsrd.com eISSN: 2456-6470

Table 3: Concentrations of Essential Metals in Cultivated Mushroom samples (mg/kg)

\begin{tabular}{|c|c|c|c|c|c|}
\hline Mushroom species & \multicolumn{5}{|c|}{ Cultivated } \\
\hline & $\mathrm{Na}$ & $\mathrm{K}$ & $\mathrm{Ca}$ & $\mathrm{Mg}$ & $\mathrm{Fe}$ \\
\hline Aph & $1,061.12$ & 701.99 & $1,841.00$ & $1,566.11$ & 772.01 \\
\hline Av & 698.60 & 196.39 & 219.69 & $1,279.00$ & 777.18 \\
\hline Range & $698.60-1061.12$ & $196.39-701.99$ & $219.69-1841.00$ & $1279.00-1566.11$ & $772.01-777.18$ \\
\hline SEM & \pm 0.19 & \pm 0.33 & \pm 0.20 & \pm 0.11 & \pm 0.12 \\
\hline
\end{tabular}

Aph-Amamita Phalaiodes Av-Amamita Verosa SEM-Standard Error Of Mean

The values were high and also in the line with values recorded for plants and mushrooms, by Tuzen $(2003)^{22}$, although higher values were reported for vegetables ${ }^{29}$.

Table 4 contains the trace metals concentrations in cultivated mushroom samples. $\mathrm{Cu}$ and $\mathrm{Cr}$ values were lower than WHO guideline levels for cultivated mushroom samples. Other trace metals concentrations were far above the WHO 1995 guideline levels for food. The data varied significantly at $(\mathrm{p}<0.05)$ among mushroom species. $\mathrm{Cu}, \mathrm{Pb}, \mathrm{Mn}$ and $\mathrm{Cr}$ showed lower trace metals concentrations in wild and cultivated mushrooms samples. Co, $\mathrm{Zn}, \mathrm{Cd}$, and Ni showed no significant differences at ( $\mathrm{p}>0.05)$ when compared with WHO 1995 permissible levels for food and vegetables.

Table 4: Concentrations of Trace Metals in Cultivated Mushroom samples (mg/ kg)

\begin{tabular}{|c|c|c|c|c|c|c|c|c|}
\hline \multirow{2}{*}{ Mush room species } & \multicolumn{8}{|c|}{ CULTIVATED } \\
\hline & $\mathrm{Cu}$ & & $\mathrm{Pb}$ & $\mathrm{Zn}$ & Q Cd & $\mathrm{Ni}$ & $\mathrm{Mn}$ & $\mathrm{Cr}$ \\
\hline Aph & 0.22 & 6.04 & 5.55 & 48.33 & 4.99 & 6.89 & 18.80 & 0.40 \\
\hline Av & 0.36 & 4.83 & hte 4.89 & na 9.33 & bl 9.88 & 22.05 & 11.60 & 1.10 \\
\hline Range & $0.22-0.36$ & $4.83-6.04$ & $\begin{array}{l}4.89-\mathrm{d} \\
5.55\end{array}$ & $\begin{array}{c}9.33-1 \text { tif } \\
48.33\end{array}$ & $\begin{array}{c}4.99- \\
9.88 \\
\end{array}$ & $\begin{array}{l}6.89- \\
22.05\end{array}$ & $\begin{array}{l}11.60- \\
18.80\end{array}$ & $\begin{array}{c}0.40- \\
1.10 \\
\end{array}$ \\
\hline SEM & \pm 0.04 & \pm 0.11 & \pm 0.19 & \pm 0.20 & \pm 00.10 & 0.70 & \pm 0.77 & \pm 0.10 \\
\hline WHO 1995 vegetables & 6.55 & 1.00 & $\begin{array}{l}5.68- \\
10.10 \\
\end{array}$ & $\begin{array}{r}18.95- \\
55.85 \\
\end{array}$ & $\begin{array}{c}0.37- \\
2.02 \\
\end{array}$ & $\begin{array}{l}3.65- \\
13.65 \\
\end{array}$ & $\begin{array}{l}50.00- \\
52.85 \\
\end{array}$ & $\begin{array}{l}10.01 \\
12.20 \\
\end{array}$ \\
\hline
\end{tabular}

Table 5 contains Concentrations of trace metals in the soils from where the two mushroom species were collected, normal agricultural soil and refuse dump soil used for mushroom cultivation. Among the two soil samples investigated, the least was Chronium $(0.19 \mathrm{mg} / \mathrm{kg}$ ) from Aphso. The highest value was Lead $(108.04 \mathrm{mg} / \mathrm{kg})$ from refuse damp soil. The values for Cu and Cd were higher than WHO guideline levels for normal agricultural soil while the less of the trace metals were within the acceptable limits. 
Table5: Concentrations ${ }^{\mathrm{a}}$ of Trace Metals in Normal Agricultural soil, Refuse dump soil and soils where mushrooms were collected.

\begin{tabular}{|c|c|c|c|c|c|c|c|c|}
\hline 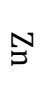 & $\bar{\sigma}$ & Z & 3 & $\Omega$ & $\Omega$ & О & $\Omega$ & $\frac{3}{\frac{3}{D}}$ \\
\hline$\underset{b}{\stackrel{V}{0}}$ & $\stackrel{\circ}{ن}$ & $\stackrel{\sim}{\infty}$ & $\underbrace{N}_{\text {vo }}$ & $\begin{array}{l}\text { No } \\
\text { N } \\
\text { ¿ }\end{array}$ & $\stackrel{\circ}{\stackrel{0}{\infty}}$ & $\stackrel{\bullet}{\circ}$ & $\stackrel{0}{\stackrel{N}{\infty}}$ & \multirow{3}{*}{ 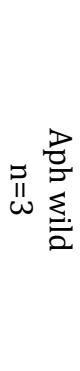 } \\
\hline $\begin{array}{l}\text { ৩ै } \\
\text { نे }\end{array}$ & نि & $\begin{array}{l}\text { No } \\
\text { Oे }\end{array}$ & $\stackrel{\sim}{\omega}$ & $\begin{array}{l}\text { 点 } \\
\text { 号 }\end{array}$ & $\begin{array}{l}\circ \\
\text { Oे }\end{array}$ & $\underset{\omega}{\omega}$ & $\begin{array}{l}\text { o } \\
\text { ò }\end{array}$ & \\
\hline $\begin{array}{l}\text { N } \\
\stackrel{N}{N}\end{array}$ & $\begin{array}{l}\text { ○ } \\
\text { 心 }\end{array}$ & $\underset{p}{\bullet}$ & $\underset{\omega}{\sim}$ & $\stackrel{\bullet}{\breve{o}}$ & 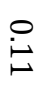 & $\begin{array}{l}\circ \\
\text { ळे }\end{array}$ & $\stackrel{0}{\stackrel{i}{N}}$ & \\
\hline $\begin{array}{l}0 \\
w \\
\dot{u}\end{array}$ & $\begin{array}{l}0 \\
\dot{\omega}\end{array}$ & $\stackrel{v}{\circ}$ & 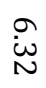 & 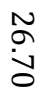 & 官 & $\stackrel{\sim}{\tilde{\omega}}$ & $\begin{array}{l}\text { o } \\
\text { ì }\end{array}$ & \multirow{3}{*}{ 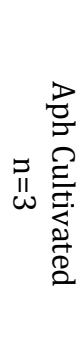 } \\
\hline$\underset{\infty}{\text { స్ }}$ & 占 & $\begin{array}{l}\omega \\
\dot{N} \\
\stackrel{N}{n}\end{array}$ & $\stackrel{\dot{\dot{O}}}{\mathrm{~N}}$ & $\begin{array}{l}\underset{N}{\omega} \\
\stackrel{\infty}{0}\end{array}$ & $\begin{array}{l}0 \\
\text { 이 }\end{array}$ & $\stackrel{\bullet}{\infty}$ & $\underset{\sim}{\stackrel{O}{A}}$ & \\
\hline ઼ֻ & $\begin{array}{l}\omega \\
\overrightarrow{0}\end{array}$ & $\begin{array}{l}\text { Na } \\
\text { gे }\end{array}$ & $\begin{array}{l}\text { No } \\
\text { o̊ }\end{array}$ & $\stackrel{N}{\stackrel{N}{0}}$ & 엄 & $\begin{array}{l}\circ \\
\text { in }\end{array}$ & $\underbrace{\circ}_{\omega}$ & \\
\hline$\stackrel{\overrightarrow{0}}{\dot{0}}$ & $\stackrel{N}{N}$ & 宛 & 卢 & $\stackrel{\bullet}{\bullet}$ & $\stackrel{\vec{\sigma}}{\circ}$ & $\stackrel{\bullet}{*}$ & $\stackrel{\sim}{\sim}$ & 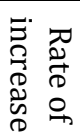 \\
\hline $\begin{array}{l}\text { ఎू } \\
\infty\end{array}$ & $\stackrel{\bullet}{\infty}$ & $\check{\omega}^{u}$ & $\stackrel{\vec{i}}{\mathrm{i}}$ & $\stackrel{N}{\stackrel{N}{\sim}}$ & $\stackrel{\triangleright}{\triangleright}$ & $\stackrel{\bullet}{\stackrel{\bullet}{\bullet}}$ & $\underbrace{\circ}_{\omega}$ & \multirow{3}{*}{ 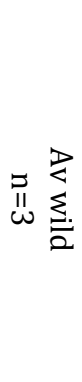 } \\
\hline $\begin{array}{l}0 \\
\infty \\
\forall\end{array}$ & 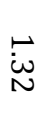 & $\stackrel{\vec{\omega}}{\vec{\phi}}$ & $\underset{\sim}{\sim}$ & $\underset{\sim}{N}$ & $\underset{\omega}{\omega}$ & மூ & $\stackrel{\circ}{0}$ & \\
\hline $\begin{array}{l}\text { Uै } \\
\text { ur }\end{array}$ & $\stackrel{\bullet}{\bullet}$ & $\begin{array}{l}\omega \\
\stackrel{\omega}{\rho}\end{array}$ & $\begin{array}{l}\text { Na } \\
\text { gh }\end{array}$ & $\underset{\sim}{\stackrel{\vec{\sim}}{0}}$ & $\begin{array}{l}\circ \\
\text { के }\end{array}$ & $\begin{array}{l}N \\
\stackrel{0}{0}\end{array}$ & $\begin{array}{l}\circ \\
\dot{\phi} \\
\dot{\phi}\end{array}$ & \\
\hline $\begin{array}{l}0 \\
\stackrel{\infty}{V}\end{array}$ & $\begin{array}{l}N \\
\text { vo }\end{array}$ & $\stackrel{+}{\stackrel{N}{0}}$ & $\underset{\omega}{\omega}$ & $\underset{\dot{\omega}}{\stackrel{P}{\vec{\omega}}}$ & $\stackrel{N}{V}$ & $\underset{\omega}{\omega}$ & 它 & \multirow{3}{*}{ 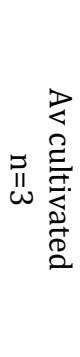 } \\
\hline $\begin{array}{l}\text { 居 } \\
\text { a் }\end{array}$ & $\underset{\sim}{\sim}$ & $\stackrel{\sim}{\stackrel{N}{v}}$ & $\stackrel{\vec{\omega}}{\vec{\omega}}$ & $\begin{array}{l}N \\
\sigma\end{array}$ & $\stackrel{\bullet}{\infty}$ & $\stackrel{\bullet}{\bullet}$ & 它 & \\
\hline $\begin{array}{l}\stackrel{\varpi}{\bullet} \\
\stackrel{\bullet}{\sigma}\end{array}$ & $\underbrace{\sim}_{\omega}$ & $\begin{array}{l}\text { Vv } \\
\infty \\
\infty\end{array}$ & $\begin{array}{l}v \\
\text { is }\end{array}$ & $\underset{\omega}{\tilde{\omega}}$ & மே & $\begin{array}{l}\vec{\omega} \\
\ddot{\bullet}\end{array}$ & $\stackrel{\bullet}{\stackrel{\bullet}{\rightleftarrows}}$ & \\
\hline$\stackrel{\stackrel{N}{N}}{ }$ & $\begin{array}{l}N \\
\tilde{u} \\
\stackrel{\sigma}{\sigma}\end{array}$ & ○ & よ் & $\begin{array}{l}N \\
\sigma \\
\sigma\end{array}$ & $\begin{array}{l}\tilde{\omega} \\
\tilde{\infty} \\
\tilde{N}\end{array}$ & $\stackrel{\bullet}{0}$ & $\stackrel{\bullet}{\omega}$ & 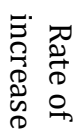 \\
\hline
\end{tabular}

\begin{tabular}{|c|c|c|c|c|c|c|c|c|}
\hline Soil/sub type & $\mathrm{Cd}$ & $\mathrm{Coz}$ & $\mathrm{Cu}$ & $\mathrm{Cr}$ & $\mathrm{Zn}$ & $\mathrm{Mn}$ & $\mathrm{Ni}$ & $\mathrm{Pb}$ \\
\hline Avso & 10.55 & 10.91 & 10.77 & 8.89 & 80.83 & 10.54 & 23.19 & 6.06 \\
\hline Aphso & 3.38 & 2.01 & 2.08 & 0.19 & 5.44 & 1.39 & 8.18 & 4.77 \\
\hline Normal agric soil & 6.04 & 1.10 & 10.62 & 2.09 & 18.83 & 2.64 & 8.66 & 66.06 \\
\hline Refuse dump soil & 17.65 & 4.44 & 15.79 & 6.44 & 22.44 & 7.89 & 18.89 & 108.04 \\
\hline WHOagric soil & $<0.0 .10$ & $2-5.6$ & $<0.10$ & $2-10$ & $60-780$ & $50-200$ & $30-75$ & $2-13$ \\
\hline WHO polluted soil & $1-3$ & $5-10$ & $50-140$ & $2-10$ & $150-300$ & $50-200$ & $30-75$ & $50-300$ \\
\hline
\end{tabular}


Table 6 shows the concentrations of trace metals in soils after harvest. There was a remarkable decrease in trace metal concentrations in soils after mushrooms harvest. The sharp difference in trace metal concentrations were significant at $\mathrm{p}<0.05$ in soils from refuse dump. This result is in line with literature reports that green vegetables could be used as bio-indicators of trace metals in polluted environment ${ }^{47}$.

Table 6: Concentrations ${ }^{\mathrm{a}}$ of trace metals in soils after harvest

\begin{tabular}{|c|c|c|c|c|c|c|c|c|}
\hline Soil types & $\mathrm{Cd}$ & $\mathrm{Co}$ & $\mathrm{Cr}$ & $\mathrm{Cu}$ & $\mathrm{Mn}$ & $\mathrm{Ni}$ & $\mathrm{Pb}$ & $\mathrm{Zn}$ \\
\hline Normal soil & 5.69 & 0.98 & 1.88 & 4.66 & 2.33 & 6.30 & 44.96 & 16.39 \\
\hline Refuse dump soil & 11.38 & 2.36 & 4.66 & 10.49 & 5.49 & 10.26 & 90.88 & 22.79 \\
\hline
\end{tabular}

Tables $7 \mathrm{a}, 7 \mathrm{~b}$ and fig 1 show the rate of increase in trace metal concentrations in mushroom after harvest.

\begin{tabular}{|c|c|c|c|c|c|c|c|c|c|}
\hline 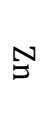 & $\sigma$ & ZZ. & 3 & 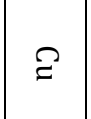 & $?$ & $\Omega$ & $\Omega$ & $\frac{3}{2}$ & \\
\hline$\stackrel{ }{\stackrel{*}{0}}$ & $\stackrel{\circ}{\text { v }}$ & $\stackrel{N}{\stackrel{N}{\infty}}$ & N & $\begin{array}{ll} & N \\
0 & 0 \\
& \text { N }\end{array}$ & $\stackrel{0}{i}$ & $\stackrel{\overrightarrow{0}}{\overrightarrow{0}}$ & $\stackrel{\circ}{\infty}$ & \multirow{3}{*}{$\vec{\omega}$} & \\
\hline $\begin{array}{l}\text { जै } \\
\text { vi }\end{array}$ & 억 & $\begin{array}{l}N \\
\stackrel{0}{0}\end{array}$ & $\stackrel{\sim}{\omega}$ & ○ & $\begin{array}{l}\circ \\
\dot{0}\end{array}$ & $\vec{\omega}$ & $\begin{array}{l}\circ \\
\text { ஸे }\end{array}$ & & 尊 \\
\hline$\stackrel{\infty}{\stackrel{N}{N}}$ & $\begin{array}{l}0 \\
\text { D } \\
\text { D }\end{array}$ & i & $\stackrel{\sim}{\omega}$ & o $\quad \vec{v}$ & $\stackrel{\circ}{\bullet}$ & $\begin{array}{l}\text { ㅇ } \\
\text { ळे }\end{array}$ & $\begin{array}{l}0 \\
\dot{i} \\
\text { N }\end{array}$ & & \\
\hline $\begin{array}{l}0 \\
w \\
u\end{array}$ & $\begin{array}{l}0 \\
\dot{\omega} \\
\omega\end{array}$ & $\stackrel{ }{\circ}$ & $\stackrel{\tilde{\omega}}{\tilde{N}}$ & $\begin{array}{ll}0 & N \\
& \sigma\end{array}$ & $\stackrel{\bullet}{\bullet}$ & $\stackrel{N}{\omega}$ & $\begin{array}{l}0 \\
\text { जू }\end{array}$ & \multirow{3}{*}{$\bar{\omega}$} & \multirow{3}{*}{ 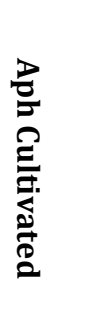 } \\
\hline సু & ஸे & Uे & $\stackrel{\dot{O}}{N}$ & $\begin{array}{l}\omega \\
N \\
0 \\
0\end{array}$ & $\begin{array}{l}\circ \\
\text { ن }\end{array}$ & $\stackrel{\bullet}{\infty}$ & $\stackrel{0}{i}$ & & \\
\hline 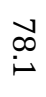 & $\begin{array}{l}\omega \\
i \\
0\end{array}$ & $\begin{array}{l}N \\
\text { ō }\end{array}$ & $\begin{array}{l}\text { No } \\
\text { o }\end{array}$ & $\stackrel{N}{\stackrel{N}{0}}$ & $\begin{array}{l}0 \\
\text { ir }\end{array}$ & $\begin{array}{l}0 \\
\text { vi }\end{array}$ & $\stackrel{\circ}{\omega}$ & & \\
\hline 官 & $\stackrel{N}{\sim}$ & 붕 & $\stackrel{\bullet}{\dot{a}}$ & $\underbrace{\bullet}_{\infty}$ & $\dot{\sigma}_{\alpha}^{+}$ & $\stackrel{\bullet}{*}$ & $\stackrel{\bullet}{\sim}$ & 芯 & 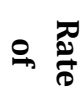 \\
\hline $\begin{array}{l}\checkmark \\
\infty\end{array}$ & $\stackrel{\bullet}{\infty}$ & $\stackrel{u}{\omega}$ & $\stackrel{\vec{i}}{\mathrm{i}}$ & $\stackrel{N}{\stackrel{N}{0}}$ & $\check{\infty}_{\infty}$ & $\begin{array}{l}\vec{尸} \\
\dot{0}\end{array}$ & $\stackrel{\circ}{w}$ & \multirow{3}{*}{$\vec{\omega}$} & \multirow{3}{*}{$\begin{array}{l}\frac{\bigotimes}{2} \\
\vdots \\
\vdots\end{array}$} \\
\hline$\stackrel{0}{\infty}$ & $\ddot{\omega}$ & $\vec{\omega}$ & $\underset{N}{\omega}$ & $\stackrel{N}{N}$ & $\vec{\omega}$ & ซึ & 웁 & & \\
\hline $\begin{array}{l}\text { गै } \\
\text { ir }\end{array}$ & 它 & $\begin{array}{l}\omega \\
\text { oे }\end{array}$ & $\begin{array}{l}\text { Na } \\
\text { gु }\end{array}$ & $\stackrel{\vec{\sim}}{\stackrel{\circ}{0}}$ & $\begin{array}{l}0 \\
\text { a }\end{array}$ & $\begin{array}{l}N \\
0\end{array}$ & $\begin{array}{l}0 \\
\dot{\phi}\end{array}$ & & \\
\hline $\begin{array}{l}0 \\
\infty \\
\forall\end{array}$ & N & $\stackrel{+}{\circ}$ & $\tilde{\omega}$ & $\stackrel{\vec{t}}{\omega}$ & $\stackrel{N}{V}$ & $\stackrel{\sim}{\omega}$ & $\stackrel{0}{\omega}$ & & \multirow{3}{*}{ 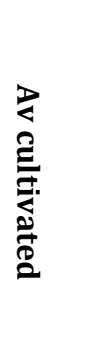 } \\
\hline $\begin{array}{l}\text { 尸 } \\
\text { ○் }\end{array}$ & $\stackrel{\sim}{\omega}$ & $\stackrel{N}{\stackrel{N}{v}}$ & $\vec{\omega}$ & $\begin{array}{l}N \\
\sigma\end{array}$ & $\stackrel{\bullet}{\circ}$ & $\vec{v}$ & $\stackrel{\bullet}{\vec{r}}$ & & \\
\hline 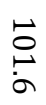 & $\underbrace{u}_{\omega}$ & $\begin{array}{l}\text { ㄱ } \\
\infty \\
\infty\end{array}$ & ir & $\underset{\omega}{N}$ & மே & $\begin{array}{l}\vec{\omega} \\
\dot{\bullet}\end{array}$ & $\stackrel{\bullet}{\dot{\oplus}}$ & & \\
\hline$\stackrel{\tilde{N}}{N}$ & $\underset{\sigma}{N}$ & $\stackrel{\circ}{\circ}$ & б. & $\begin{array}{l}\text { Na } \\
\sigma \\
\sigma\end{array}$ & $\stackrel{\sim}{\omega}$ & iै & $\stackrel{\omega}{\omega}$ & 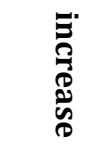 & 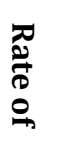 \\
\hline
\end{tabular}

Rate of Increase $=\mathrm{C}_{\mathrm{M}} / \mathrm{W}_{\mathrm{m}}$, where $\mathrm{Cm}=$ Concentrations trace metals in cultivated mushrooms. Wm=Concentrations of trace metals in wild mushrooms. 
International Journal of Trend in Scientific Research and Development (IJTSRD) @ www.ijtsrd.com eISSN: 2456-6470

Table: $7 \mathrm{~b}$ Rate of increase in trace metal concentrations in mushrooms after harvest.

\begin{tabular}{|c|c|c|}
\hline Metals & Amanita phalaoides & Amanita verosa \\
\hline $\mathrm{Cd}$ & 1.21 & 1.31 \\
\hline $\mathrm{Co}$ & 1.17 & 1.97 \\
\hline $\mathrm{Cr}$ & $4.60^{\mathrm{a}}$ & 2.38 \\
\hline $\mathrm{Cu}$ & 1.78 & $2.66^{\mathrm{c}}$ \\
\hline $\mathrm{Mn}$ & 1.64 & 1.66 \\
\hline $\mathrm{Ni}$ & 1.95 & 0.01 \\
\hline $\mathrm{Pb}$ & $2.78^{\mathrm{b}}$ & 2.54 \\
\hline $\mathrm{Zn}$ & 1.05 & 1.22 \\
\hline
\end{tabular}
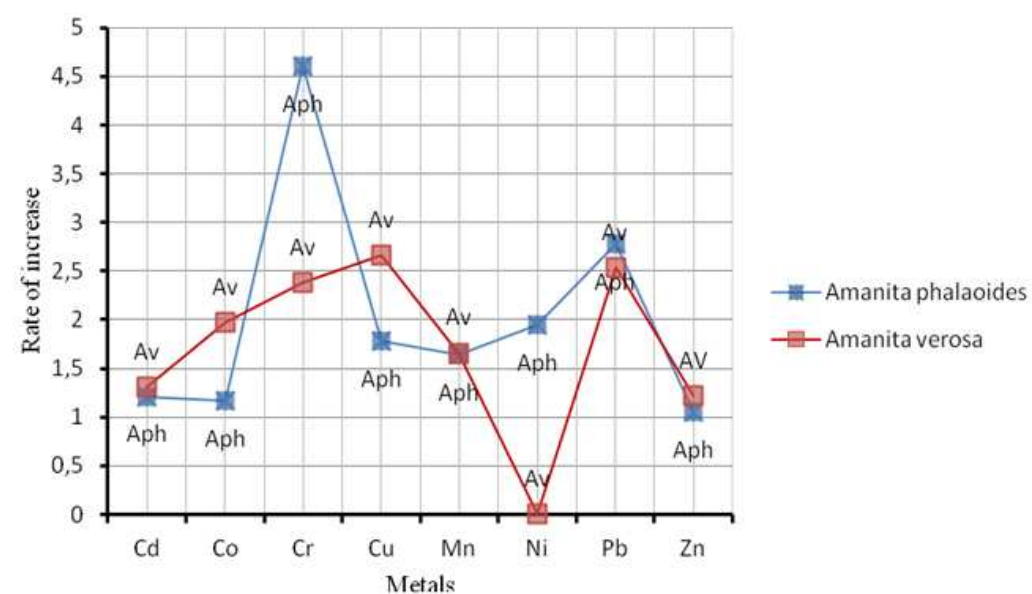

- Amanita verosa

Fig 1 showed the graphical representation of rate of increase in cultivated mushrooms versus wild mushrooms. Aph absorbed $\mathrm{Cr}$ in appreciable quantities while $\mathrm{Ni}$ has the lowest value in Av see fig 1 . Rate of increase in $\mathrm{Cd}, \mathrm{Co}, \mathrm{Cr}, \mathrm{Cu}, \mathrm{Mn}, \mathrm{Pb}$ and $\mathrm{Zn}$ were higher in mushrooms collected from forest soil near industrial sites and heavy traffic.

Table 8 contains the bioaccumulation factors/Accumulation coefficients of mushrooms cultivated on agricultural soil. Cobalt was highest bio- accumulated in Av while the rest of the trace metals were bio-accumulated in the following order: $\mathrm{Cu}>\mathrm{Zn}>\mathrm{Mn}>\mathrm{Cr}>\mathrm{Cd}>\mathrm{Ni}>\mathrm{Pb}$.

Table 8: Accumulation Coefficients of mushrooms grown on Agricultural soil (Amanita phaloides and Amanita verosa)

\begin{tabular}{|c|c|c|c|c|c|c|}
\hline Metals & $\begin{array}{c}\text { Normal Agricultural soil } \\
\text { metal concentration }\end{array}$ & $\begin{array}{c}\text { Amanita } \\
\text { phalaoides }\end{array}$ & $\begin{array}{l}\text { Acc. } \\
\text { Coeff. }\end{array}$ & $\begin{array}{c}\text { Normal Agricultural soil } \\
\text { metal concentration }\end{array}$ & Av & $\begin{array}{l}\text { Acc. } \\
\text { Coeff. }\end{array}$ \\
\hline $\mathrm{Cd}$ & 5.69 & 10.89 & 1.91 & $\infty 8$ & 18.36 & 3.23 \\
\hline Co & 0.98 & 10.08 & $10.29 b$ & 0.98 & 16.89 & $17.24 \mathrm{a}$ \\
\hline $\mathrm{Cr}$ & 1.88 & 7.33 & 3.90 & 1.88 & 10.01 & 5.33 \\
\hline $\mathrm{Cu}$ & 4.66 & 15.88 & 3.41 & 4.66 & 33.69 & $7.23 c$ \\
\hline $\mathrm{Mn}$ & 2.33 & 8.96 & 3.85 & 2.33 & 6.67 & 2.86 \\
\hline $\mathrm{Ni}$ & 6.30 & 13.77 & 2.19 & 6.30 & 18.99 & 3.01 \\
\hline $\mathrm{Pb}$ & 44.96 & 50.76 & 1.13 & 44.96 & 71.66 & 1.59 \\
\hline $\mathrm{Zn}$ & 16.39 & 88.95 & 5.43 & 16.39 & 69.88 & 4.26 \\
\hline
\end{tabular}

a,b,c are subscripts showing significant differences at $\mathrm{p}<0.05$.

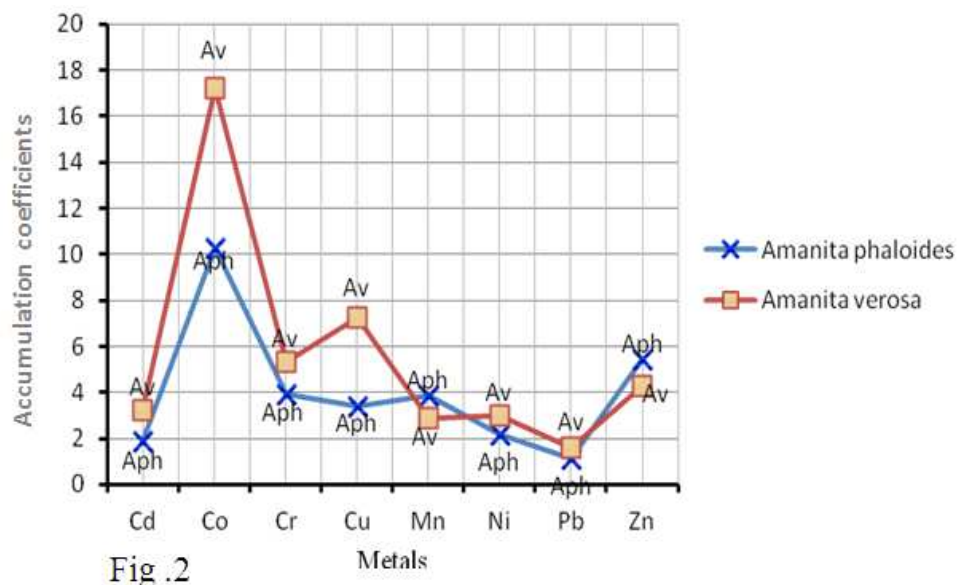


International Journal of Trend in Scientific Research and Development (IJTSRD) @ www.ijtsrd.com eISSN: 2456-6470

BIOACCUMULATION FACTORS/ACCUMULATION COEFFICIENTS OF TRACE METALS $\mathrm{k}_{\mathrm{a}}=\mathrm{Cm} / \mathrm{Cs}$ where Cm= concentrations of trace metal in mushroom, $\mathrm{Cs}=$ concentrations of trace metals in soil used for cultivation.

Table 9 contains the bioaccumulation factors of mushrooms cultivated on refuse dump soil.

Table9: Accumulation coefficients of Mushrooms cultivated on refuse dumps soil (Amanita phaloides and Amanita veros

\begin{tabular}{|c|c|c|c|c|c|c|}
\hline Metals & Refuse dump soil & Amanita phalaoides & Acc. Coeff. & Refuse dump soil & Av & Acc. Coeff. \\
\hline $\mathrm{Cd}$ & 11.38 & 22.09 & 2.02 & 11.38 & 28.44 & 2.50 \\
\hline $\mathrm{Co}$ & 2.36 & 25.44 & 10.78 & 2.36 & 23.17 & 9.82 \\
\hline $\mathrm{Cr}$ & 4.66 & 33.19 & 7.12 & 4.66 & 54.67 & $11.73 \mathrm{c}$ \\
\hline $\mathrm{Cu}$ & 10.49 & 63.11 & 6.02 & 10.49 & 69.31 & 6.61 \\
\hline $\mathrm{Mn}$ & 5.49 & 19.46 & 3.54 & 5.49 & 15.88 & 2.89 \\
\hline $\mathrm{Ni}$ & 10.26 & 23.68 & 2.31 & 10.26 & 35.10 & 3.42 \\
\hline $\mathrm{Pb}$ & 90.88 & 184.32 & 2.03 & 90.88 & 166.38 & 1.83 \\
\hline $\mathrm{Zn}$ & 2.79 & 189.44 & $67.90 \mathrm{~b}$ & 2.79 & 201.46 & $72.21 \mathrm{a}$ \\
\hline
\end{tabular}

a,b,c are subscripts showing significant differences at $\mathrm{p}<0.05$.

Zn was highest in Amanita verosa(72.21) followed by Amanita phalaoides(67.90) cultivated in refuse dump soil. Heavy metals contents of all analyzed mushrooms were higher than those previously reported in literature.

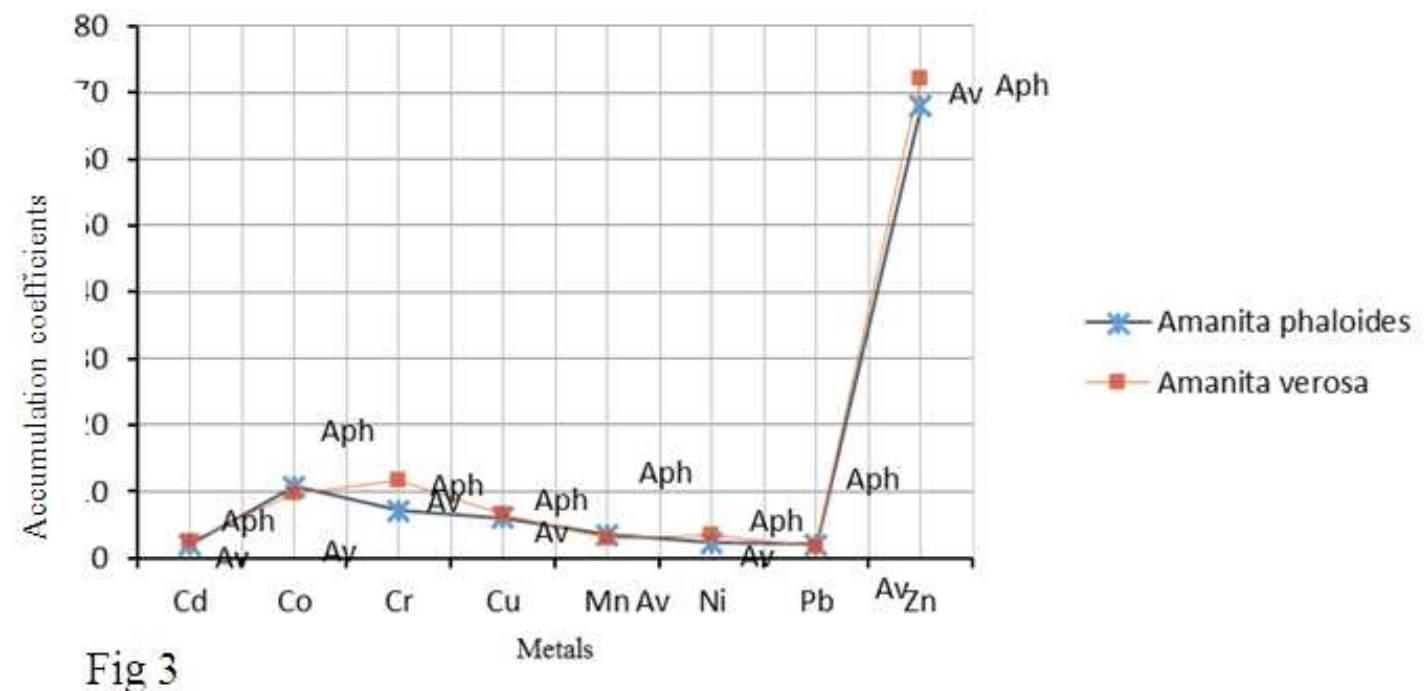

Fig 3 shows graphical representation of bioaccumulation factors of mushrooms cultivated on refuse dump site(Aph in blue and $\mathrm{Av}$ in red). Lead have the least value while $\mathrm{Zn}$ have the highest value.

Thus this observation was supported by various authors ${ }^{24,31,37}$. It was noted that Plants translocate larger quantities of metals to their leaves than to their fruits or seeds ${ }^{17,19}$. The high values of lead (Pb), cobalt (Co), candium (Cd) and Nickel (Ni) obtained are the serious health concern observed among the mushroom species. This poses a serious health risk among the people that excessively consume these mushrooms. Also, Isildak et al;(2001) ${ }^{21}$ reported that mushroom species in relation to heavy metal bioaccumulation are affected by substrate composition, age of the fruiting bodies and distance from the source of pollution. This is an indication that the metal uptake can be controlled when these mushrooms are grown under a disease free environment.

\section{DISCUSSION}

Proximate composition, mineral content and anti-nutritional factors are indices of nutritional qualities. The values obtained from studied mushrooms clearly indicate that these mushrooms have high nutritional qualities and are safe for human consumption. For example, crude protein (CP) (8.5024.18) $\%$ are fairly comparable with those of legumes (Cowpea $19.06 \%$, Beans $20.80 \%$, Groundnut $23.05 \%$ ) and meat muscle (22.00\%). Ash contents $3.26 \%-14.33 \%$ are indications of high mineral contents of mushrooms. Lipid (fat/oil) $1.00 \%-6.68 \%$ are indications that mushrooms are excellent dietary food for diabetic and coronary heart disease patients. From the present analytical information, it is conceivable that these mushrooms (Amanita phalaoides, and Amanita verosa) hold tremendous promise in narrowing the protein and mineral supply deficiency prevalent in several developing countries of Africa (Ijioma et al 2015) ${ }^{27}$.
Carbohydrates (CHO) contents of 32-35.40 \% explained why some mushroom species are used locally as binder, bulking agent (in melon cake-a local snack) or a thickener in soups. This implies that mushrooms can function effectively in low fat diet such as those required by patients with cardiovascular diseases, obesity etc (Okhuoya et al., 2010)14.

The vitamin $\mathrm{C}$ contents of mushroom samples were detectable at levels ranging from $0.01 \%$ to $0.37 \%$. However, the values of vit $\mathrm{C}$ determined shows that the mushroom species are not reliable sources of Vitamin C, although they can make important contribution to diet.

The mean values for anti-nutritional factors of studied mushrooms ranged as follows: phytate $0.26 \mathrm{mg} / 100 \mathrm{~g}$, cyanides $0.16 \mathrm{mg} / 100 \mathrm{~g}$ ) and tannins $0.31 \mathrm{mg} / 100 \mathrm{~g}$ ). The 
mean values for anti-nutritional factors are not up to safe limit $(0.36 \mathrm{mg} / 100 \mathrm{~g})$ in mushrooms and $1.00 \mathrm{mg} / 100 \mathrm{~g}$ in food where the guidelines were higher. This shows that the toxic effect of these substances may not be experienced by the consumers since there is further destruction of these substances during cooking. Similar values $(0.36 \mathrm{mg} / 100 \mathrm{~g})$ have been reported for phytates in some mushrooms and food (Aletor 1995) ${ }^{33}$. These values are not higher than those reported for cowpea $(0.36 \mathrm{mg} / 100 \mathrm{~g})$ and soyabean $(0.80$ $\mathrm{mg} / 100 \mathrm{~g})^{30}$. Phytates chelate mineral elements such as $\mathrm{Ca}$, $\mathrm{Mg}, \mathrm{Fe}$ and $\mathrm{Zn}$ which renders the elements unavailable for absorption, (Gropper 2009) ${ }^{37}$. Phytates also inhibit the activities of digestive enzymes: amylase, pepsin and pancreatin, (Griffiths,1979) ${ }^{36}$.

The mean cyanide contents $0.16 \mathrm{mg} / 100 \mathrm{~g}$ of these mushroom species are close to $0.36 \mathrm{mg} / 100 \mathrm{~g}$ obtained by Kalac et al (2000) ${ }^{27}$. This factor can be eliminated through branching and cooking prior to consumption ${ }^{25}$. Tannins comprised of a heterogeneous group of plant polyphenols which are capable of interacting with proteins. Tannins are high molecular weight compounds (mol. Wt. 500-5000) containing sufficient phenolic hydroxyl groups to permit the formation of stable cross-links with proteins ${ }^{26}$. However, one major problem encountered with polyhenol research remains that lack of a standard quantitative method for the analysis of tannins that would be suitable for a wide range of seed, forage, crops and food products under varying experimental conditions.

Tannins form complexes with proteins, carbohydrate and other polymers in foods and certain metal ions, under suitable conditions of concentration and $\mathrm{pH}^{18}$. The tendency of tannin to complex with proteins more than carbohydrates and other food polymers is attributed to the strong hydrogen bond affinity of the carbon, oxygen of the peptide group. Not much has been reported on the tannin contents of mushrooms in Nigeria ${ }^{25}$.However, the mean values were low when compared with $1.0 \mathrm{mg} / 100 \mathrm{~g}$ in $(\mathrm{WHO1995})^{44}$ guideline levels.

The mean phytic acid contents $0.31 \mathrm{mg} / 100 \mathrm{~g}$ were low for wild and cultivated mushroom species when compare with $1.00 \mathrm{mg} / 100 \mathrm{~g}$ (WHO) ${ }^{44}$ guideline levels. Phytates are a complex class of naturally occurring compounds. The knowledge of phytic acid began with the isolation of small particles or grains which use not starch from the seeds of various plants ${ }^{23}$.

Phytic acid is called hexaphospherylated myo-inositol or myo-inositol hexaphosphoric acid or more scientifically 1,2 , $3,4,5,6$,-hexalone (dihydrogen phosphate) myo-inositol. It is a storage compound ubiquitously distributed in the plant kingdom. It is an anti-nutritional factor impairing the utilization of other nutrients such as proteins and minerals. Phytate has been shown to bind zinc and other minerals. Also zinc and other mineral have been demonstrated to improve the utilization of proteins ${ }^{27}$. Nigerians consume and utilize plant foods as their source of nutrients, so it is important to determine the phytate content of plant foods mostly consumed by majority of the population. The ability of phytic acid to form complexes with metals is one of the main nutritional concerns. These complexes present in metal absorption and availability. The effects of phytic acid on mineral bioavailability are rather conflicting and confusing because there are other interacting components of food such as fibre ${ }^{46}$.

The concentrations of essential metals in table 4 indicates that these mushroom species are good sources of these mineral elements and could provide up to $50 \%$ of the recommended daily allowances for these elements. The mean values were also higher than those reported in literature. For example: cowpea ( $\mathrm{Na} 277.33 \mathrm{mg} / \mathrm{kg}$ ), vegetables $(252.67 \mathrm{mg} / \mathrm{kg}$, mushrooms $(772.42 \mathrm{mg} / \mathrm{kg})$ but lower than the values reported for fish $(1,061.12 \mathrm{mg} / \mathrm{kg})$, snails $(1,566.11 \mathrm{mg} / \mathrm{kg}$ ) and meat $(1,279.00 \mathrm{mg} / \mathrm{kg}$ ) (Aletor, $1995)^{33}$.

Concentration of Sodium varies in species parts which was similar observation made by Isiloglu et al; $(2001)^{21}$. Calcium (ca) was dominant in the veil part of all the five species showing the attractiveness and the physical support of the veil structure. Also, adequate calcium has contributed immensely to the succulent nature of the veil. These values were higher than values reported for vegetables ${ }^{31}$.

The zinc contents ranged from 50.88-68.56 mg/kg for wild and cultivated respectively are slightly above permissible level of $50 \mathrm{mg} / \mathrm{kg}$ (WHO, 1995) ${ }^{44}$. Zinc is wide spread in living organisms due to its biological significance. The levels reported here are in agreement with values reported by Turkekul et al., (2004) ${ }^{25}$ and Tuzen (2003) ${ }^{22}$. The cadmium content ranged from $2.25 \mathrm{mg} / \mathrm{kg}$ in Schizophyllum commune to $4.88 \mathrm{mg} / \mathrm{kg}$ in V.volvacea. The levels of cadmium in edible mushrooms were higher than the WHO permissible limit (WHO, 1995) ${ }^{44}$. Cadmium contents of mushroom samples in the literature have been reported to be in the ranges: 0.81$7.50 \mathrm{mg} / \mathrm{kg}$ (Ayodele et al. 2007) ${ }^{17}$.The lead level ranged from 1.25 to $1.88 \mathrm{mg} / \mathrm{kg}$ for Pleurotus squarrosollus and Aricularia auricula. Lead contents of mushroom samples in the literature have been reported to be in the ranges: 0.75 $7.77 \mathrm{mg} / \mathrm{kg}$ (Isilogu et al 2001) ${ }^{21}, 0.40-2.80 \mathrm{mg} / \mathrm{kg}$ (Fasola et al., 2007) ${ }^{23}, 1.43-4.17 \mathrm{mg} / \mathrm{kg}$ (Tüzen, 2003) ${ }^{22}, 0.800$ $2.700 \mathrm{mg} / \mathrm{kg}$ (Isildak et al., 2004), $0.82-1.99 \mathrm{mg} / \mathrm{kg}$ (Zheng et al., 2003) ${ }^{28}$, and $0.9-2.6 \mathrm{mg} / \mathrm{kg}$ (Hitivani., 2003), respectively. The lead values for wild mushroom species were similar with those found in literature while cultivated mushrooms on refuse dump soil gave a higher value.

Lead like Cadmium has no beneficial role in human metabolism, producing progressive toxicity. Lead accumulates in bones, and it can take in place of calcium. Lead creates health disorders such as sleeplessness, tiredness, hearing, and weight loss. Minimum and maximum concentration of $\mathrm{Cu}$ accumulated by the mushrooms was 1.55 and $1.86 \mathrm{mg} / \mathrm{kg}$ respectively, with Aricularia auricular accumulating the highest $\mathrm{Cu}$ concentration of $1.86 \mathrm{mg} / \mathrm{kg}$. Isildak et al. (2004) $)^{5}$ reported a $\mathrm{Cu}$ concentration of $107 \pm$ $8.5 \mathrm{~g} / \mathrm{g}$ in wild growing Agaricus biosporus from the middle black sea region of Turkey.

Tables 4-8 showed higher concentration levels of $\mathrm{Cr}, \mathrm{Zn}, \mathrm{Ni}$, $\mathrm{Cu}, \mathrm{Mn}, \mathrm{Co}, \mathrm{Cd}$ and $\mathrm{Pb}$ predominantly noticed in cultivated mushrooms from the refuge dump near a busy road. This could be attributed to exposure of the mushrooms to atmospheric deposition of dust and traffic pollution in the urban places most especially where there are heavy traffic of vehicles. Ugwu et al reported that air pollution may pose a threat to green plants especially during the hazy and harmattan season and along roadside ${ }^{11}$ 
Furthermore, as the vehicle plow through dusty roads, they cause pre-released lead and other toxic metals like nickel, cd and $\mathrm{Cr}$ to be washed back into the air. Fumes from automobile exhaust would accumulate more on exposed food such as mushrooms from high traffic density (go-slow) than in low traffic conditions. Reports have it that vehicles contributes enormously to the level of metallic compounds in food sample which depends on the level of traffic in such location $^{18}$

Also particulate air pollution from industries, more vehicles, waste disposal activity, incineration, chemical plants, metal production and PVC factories, oil refineries etc contribute to release of toxic contaminants in the urban areas. Anoliefo et al $(2003)^{20}$ reported that particular air pollution and vehicles are the main causes of heavy metal contamination in urban areas, ${ }^{21}$.

Similar works show that traffic volume, industrial activities and intensity of human activities are sources of soil contaminant in the urban areas ${ }^{24}$. Some works have it that rapid urbanization, unorganized industrialization and increased use of automobile have contributed to the elevated levels of heavy metals in the urban environment ${ }^{25}$. Akerendolu(1989) ${ }^{29}$ also reported that the exceptional lead concentration is due to the heavy lead of contaminated dust in the air of a very crowded city and firms from automobiles ${ }^{27}$. Dust mobilization done to automobiles has been estimated to be $6.5 \mathrm{~g} / \mathrm{vehicle} \mathrm{km}$ for paved roads and $61.5 \mathrm{~g} /$ vehicle $\mathrm{km}$ for unpaved roads in Nigeria. Compared to only $0.1 \mathrm{~g} /$ vehicle $\mathrm{km}$ for streets in London, England 28,29 .

The lower concentration levels of $\mathrm{Pb}, \mathrm{Cd}, \mathrm{Ni}, \mathrm{Cr}, \mathrm{Cu}$, and $\mathrm{Co}$ in wild mushrooms from the rural areas where vehicular and industrial activities are less could be taken as background levels of these contaminants in the soil for the mushrooms.

The data were analyzed statistically to determine whether there were significant differences between the concentration levels of $\mathrm{Pb}, \mathrm{Cd}, \mathrm{Ni}, \mathrm{Cr}, \mathrm{Mn}, \mathrm{Zn}$, $\mathrm{Co}$ and $\mathrm{Cu}$ in cultivated mushrooms from the contaminated soil and wild (natural habitat). Mean concn levels of $\mathrm{Pb}$ and $\mathrm{Cd}$ in wild mushrooms from the sub- urban and rural areas were significantly different $(\mathrm{p}<0.05)$,a sub-urban commercial and fairly industrialized town Nnewi (urban/industrial influence). Most of the lead and Cd concentration levels in the mushrooms are from the soil where the mushrooms were harvested since most of the mushrooms sold in the urban areas were actually from the rural areas where industrial and vehicular activities were less. This shows that urban deposition had significant influence on the lead and Cd levels in wild mushroom samples compare with levels in cultivated.

The mean concentration levels of $\mathrm{Ni}, \mathrm{Cr}, \mathrm{Mn}$, and $\mathrm{Cu}$ in wild from the semi-urban areas were significantly higher $(\mathrm{P}<0.05)$ than the mean concentration levels determined in the samples collected from the rural areas. These differences could be attributed to vehicles emission, particulate air pollution from industries, dust from busy roads due to high traffic density, crowded areas and other urban industrial emissions whereas the presence of these contaminants in the samples from the rural areas can be linked to background levels in the soil where the mushrooms were grown. Reports have it that the content of $\mathrm{Mn}$ in food depends on the Manganese content of the soil where the plants were grown ${ }^{30}$. Other works show that although $\mathrm{Ni}, \mathrm{Cr}$ and $\mathrm{Cu}$ occur naturally in the soil through volcanic emission, weathering of soil and rock, they can easily be released into the air through burning of fossil fuels and plastics containing particularly acrylonitriles ${ }^{30,31}$

Among metals determined, Chromium value was very low in forest soil $(0.19 \mathrm{mg} / \mathrm{kg})$ where Aph was collected, and leaf litter $(0.03 \mathrm{mg} / \mathrm{kg})$. The highest value was that of Lead $(108.04 \mathrm{mg} / \mathrm{kg}$ ) from refuse dump soil. $\mathrm{Cu}$ and $\mathrm{Cd}$ were higher than WHO guideline levels for normal agricultural soil in soils/substrates showing high levels of contamination of soils and substrates by $\mathrm{Pb}, \mathrm{Cu}$ and $\mathrm{Cd}$ in the sampled areas.

It was observed that values obtained in veils were higher than values obtained in stems in all the species studied. There were significant differences at $(\mathrm{p}<0.05)$ between the values of stems and veils. $\mathrm{Cd}(3.18 \mathrm{mg} / \mathrm{kg})$ for Amanita phalaiodes was above WHO 1995 guideline levels vegetables, while Co $(1.01 \mathrm{mg} / \mathrm{kg})$ for Aphv and Avv for cultivated mushrooms were close to WHO guideline levels.

Among metals determined, $\mathrm{Mn}$ is the most bio-accumulated while the rest are bio-accumulated in the following order $\mathrm{Zn}>\mathrm{Ni}>\mathrm{Cd}>\mathrm{Pb}>\mathrm{Co}>\mathrm{Cu}>\mathrm{Cr}$. Among the mushroom species Aph show the highest bio-accumulation of $\mathrm{Mn}$ and $\mathrm{Zn}$. Other specie with high bio-accumulation of metals is Av.

Among metals determined, $\mathrm{Cr}$ is the most bio-accumulated while the rest are bio-accumulated in the following order $\mathrm{Mn}>\mathrm{Zn}>\mathrm{Co}>\mathrm{Cd}>\mathrm{Pb}>\mathrm{Ni}>\mathrm{Cu}$. Among the mushroom species Aph shows the highest bio-accumulation of Cr. Other specie with high bio-accumulation of metals is Av.

Among metals determined, $\mathrm{Mn}$ is the most bio-accumulated while the rest are bio-accumulated in the following order $\mathrm{Co}>\mathrm{Pb}>\mathrm{Zn}>\mathrm{Cu}>\mathrm{Ni}>\mathrm{Cd}>\mathrm{Cr}$. Among the mushroom species $\mathrm{Av}$ shows the highest bio-accumulation of $\mathrm{Mn}$ in Nnewi (urban/industrial influence).

Values obtained for $\mathrm{Mn}$, $\mathrm{Co}$ and $\mathrm{Pb}$ were higher when compared with other trace metals within Nnewi. Also when compared with values obtained in rural areas with little or no industrial activities. The values were still higher which support the literature reports that higher industrial activities increase availability of toxic metals in environment ${ }^{31}, 33$. Heavy metals, such as cadmium, copper, lead, chromium and mercury, are important environmental pollutants, particularly in areas with high anthropogenic pressure. Their presence in the atmosphere, soil and water, even in traces can cause serious problems to all organisms, and heavy metal bioaccumulation in the food chain, moreover, it can be highly dangerous to human health. Heavy metals enter the human body mainly through two routes namely: inhalation and ingestion, ingestion being the main route of exposure to these elements in human population. Heavy metals intake by human populations through food chain has been reported in many countries. Soil threshold for heavy metal toxicity is an important factor affecting soil environmental capacity of heavy metal and determines heavy metal cumulative loading limits. For soil-plant system, heavy metal toxicity threshold is the highest permissible content in the soil (total or bioavailable concentration) that does not pose any phytotoxic effects or heavy metals in the edible parts of the crops does not exceed food hygiene standards. Factors affecting the thresholds of dietary toxicity of heavy metal in 
soil-crop system include: soil type which includes soil pH, organic matter content, clay mineral and other soil chemical and biochemical properties; and crop species or cultivars regulated by genetic basis for heavy metal transport and accumulation in plants. In addition, the interactions of soilplant root-microbes play important roles in regulating heavy metal movement from soil to the edible parts of crops. Agronomic practices such as fertilizer and water managements as well as crop rotation system can affect bioavailability and crop accumulation of heavy metals, thus influencing the thresholds for assessing dietary toxicity of heavy metals in the food chain.

This investigation also revealed the need for public awareness on mushrooms and their bioaccumulation ability especially in areas with frequent pollution like crude oil pollution in the Niger Delta area of Nigeria. Further research is needed to find out the variations in metal uptake by different mushroom species, and the site-specific risk assessment guidelines to highlight and minimize the potential health risks of ingesting mushrooms containing high levels of heavy metals.

\section{CONCLUSION}

Some of the trace metals studied are good in maintaining a balance health condition, their tendencies of being toxic have given rise to this research work. This research is meant to stand as a guide to other researchers, nutritionists, and agriculturists who seek information on the level of trace metals in mushrooms grown in the study area and also give anyone who has the opportunity of laying hands on this research the knowledge of the effects associated with the intake of these mushroom species. Perhaps the most important conclusion that may be drawn from this study, is that since mushrooms tend to absorb and accumulate toxic metals, then mushrooms should not be cultivated in that area or the government should set up an environmental protection committee in the area to stop refuse dumping in the area. The author strongly recommends that people living in this area should not eat large quantities of these mushrooms harvested from this area, so as to avoid excess accumulation of heavy metals in the body. Thus regular monitoring of these toxic metals from the refuse and effluents dumpsite, in mushrooms and other food materials are essential, to prevent their excessive build-up in the food chain.

This investigation also revealed the need for public awareness on mushrooms and their bioaccumulation ability especially in areas with frequent pollution like crude oil pollution in the Niger Delta area of Nigeria. Further research is needed to find out the variations in metal uptake by different mushroom species, and the site-specific risk assessment guidelines to highlight and to minimize the potential health risks of ingesting mushrooms containing high levels of heavy metals.

\section{REFERENCES}

[1]. Agrahar- Murugkar, D., Subbulakshmi, G. (2005). Nutritional value of edible wild mushrooms collected from the Khasi hills of Meghalaya. Food Chem., 89:599- 603.

[2]. Emuh, F.N.(2009). Bioremediation Potentials of White Rot Fungi in the Reclamation of crude oil Polluted Soil.
Ph.D. Thesis submitted to Postgraduate Studies, Delta State University, Abraka, Nigeria,180 p.

[3]. Hamman, S. (2004). Bioremediation capability of white rot fungi. B- 1570, Review Article, Spring 2004. Int.J.Curr.Microbiol.App.Sci (2014) 3(10) 52-5757.

[4]. Hitivani, N., Mecs, L. (2003). Effects of certain heavy metals, on the growth, dye decolouration and enzyme activity of Lentinula edodes. Ectoxicol. Environ. Safety 55(2): 199203.

[5]. sildak, O. Turkekul, L. Elmastas and Tuzen, M. (2004). Analysis of heavy metals in some wild-grown edible mushrooms from the middle Black sea region, Turkey. Food Chem., 86: 547-552.

[6]. Jonathan, S.G. (2002). Vegetative growth requirements and antimicrobial activities of some higher fungi in Nigeria. Ph.D thesis, University of Ibadan, Ibadan, Nigeria.

[7]. Juhasz, A.L., Naidu, R. (2002). Bioremediation of high molecular weight polycyclic aromatic hydrocarbon: a review of microbial degradation of Benzo [(a)] pyrene. Int. Biodeterioration Biodegrad., 45:57 88.

[8]. Kalac, P., Svoboda, L. (2000). A review of trace element concentrations in edible mushrooms. Food Chem. 69: 273281.

[9]. Sasek, V. (2003). Why Myco-remediation has not come into practice. In: Problems and solution. V. Sasek, Glaser, J.A., Baveye, P., (Ed.). Dordrecht. The Netherlands. Kluwer Academic publishers. 247 -266.

[10]. Schliephake, K., Baker and, W. I., Longergan, G.T.(2003). Decolorization of industrial wastes and degradation of dye water. In: Fungal biotechnology in Agricultural, food and Environmental applications.

[11]. Akpaja, E.O., Isikhuemhen, O.S. and Okhuoya, J.A. (2003). Ethnomycology and usage of edible and medicinal mushrooms among the Igbo people of Nigeria. International Journal of Medicinal Mushrooms 5:313-319.

[12]. Wasser, S., Berreck and, M. Haselwandler,K.(2003). Radiocesium contaminants of wild growing mushroom in Ukraine. Int. J.Med. Mushrooms, 5: 6186

[13]. Stamets, P. (2005). Mycelium running. How mushroom can help save the world, $1^{\text {st }}$ Edn. Ten Speed Press, Berkeley/Toronto. 339.

[14]. Okhuoya, J.A, Akpaja, E.0, Osemwegie, 0.0, Oghenekaro, A. O, Ihayaere, C.A; (2010) Nigerian Mushroom: Underutilized Non-wood Forest Resources. J.A Sci. Environ. Manage. 14:46-54.

[15]. Akpaja, E.O., Isikhuemhen, O.S. and Okhuoya, J.A. (2003). Ethnomycology and usage of edible and medicinal mushrooms among the Igbo people of Nigeria. International Journal of Medicinal Mushrooms 5:313-319

[16]. Adebayo, J.G., Banjo, N.O. and Abikoye, E.T. (2009). Evaluation of yield of oyster mushroom (Pleurotus pulmonarius) grown on cotton waste and cassava peel. African Journal of Biotechnology 8(2):215-218.

[17]. Ayodele, M.S. and Okhuoya, A.J.( 2007). Cultivation studies on Psathyrella atrounmbonata Pegler a 
Nigerian edible mushroom on different agro-industrial wastes. International Journal of Botany 3(4):394-397.

[18]. Erguven, M., O. Yilmaz, M. Deveci, N. Aksu, F. Dursun, M. Pelit, and N. Cebeci. (2007). Mushroom poisoning. Indian Journal of Pediatrics74(9): 847-52.

[19]. Soylak, M., Saracoglu, S., Tüzen, M., Mendil, D. (2005). Determination of trace metals in mushroom samples from Kayseri, Turkey. Food Chem., 92: 649652.

[20]. Anoliefo, G.O., Isikhuemhen, O.S., Ohimain, E. (2003). Phytoassessment of soil polluted with Forcados blend crude oil exposed to a white- rot fungus, Pleurotus tuberregium (Fr.) Singer. Proceedings of the National Conference on Environmental Science and Technology. 8-9 September, 2002, Greensboro.43-59.

[21]. Isiloğlu M, Yilmaz F, Merdivan M. (2001). Concentrations of trace elements in wild edible mushrooms. Food chemistry 73:169-175.

[22]. Tüzen, M.( 2003). Determination of heavy metals in soil, mushroom and plant samples by atomic absorption spectrometry. Microchem. J., 74: 289297.

[23]. Fasola, R. T., Golagade, S.J. and Fasidi, O.I.( 2007). Nutritional requirements of Volvariella speciosa (Fr. ex Fr.) Singer, a Nigerian edible mushroom. Food Chemistry 100:904- 908.

[24]. Sesli, E., Tüzen, M., Soylak, M.(2008). Evaluation of trace metal contents of some wild edible mushrooms from Black sea region, Turkey. J.azard. Mater.,160: 462 467.

[25]. Turkekul, I., Elmastas, M., Tuzen, M. (2004). Determination of iron, copper, manganese, zinc, lead and cadmium in mushroom samples from Tokat, Turkey. Food Chem., 84: 389392.

[26]. Emeka Okafor .C. (2004).An entrepreneur residence in New York; A new Africa and Africans with a focus on entrepreneurship innovation, technology, practical remedies and other self sustaining activities. Retrieved through internet on Monday July,2015

[27]. Ijeoma, B. C; Ihediohanma, N. C; Onuegbu, N. C and Okafor, D.C (2015):Nutritional composition and some anti-nutritional factors of three edible mushroom species in south eastern Nigeria: European Journal of Food Science and Technology vol.3,No.2, 57-63, published by European Centre for Research Training and Development(www.eajournals.org).Retrieved on May,2015.

[28]. Zheng, Y. M. Chen, T.B. Wu, H. T; Chen, H; Zhou, J. L; Luo, J. F and Huang, Z.C. (2003): The Spatial Structure and Contents Distribution of Nickel in Soils of Beijing Suburb. Acta Geographical sinicg 58: 470-476.

[29]. Akeredolu, F. (1989): Atmospheric Environmental Pollution, 23:783-792.

[30]. MC Grath,D and MC Cormack,R.J.(1999).The significance of heavy metals and organic micro pollutants in soil.Rural Envirn. Ser.23 Johnstown Castle Res. Centre, Wexford,Ireland. p278.

[31]. Long XX, Yang XE, Ni WZ, Ye ZQ, He ZL, Calvert DV, Stoffella JP.(2003) Assessing zinc thresholds for phytotoxicity and potential dietary toxicity in selected vegetable crops. Commun Soil Sci Plant Anal. 2003;
34(9 \& 10):1421-1434. doi: 10.1081/CSS-120020454. [Cross Ref]

[32]. Aletor, V. A (1990). Anti - nutritional factors in some Nigeria foodstuffs. Herbage by products, Crop residues and browse plants. A monograph prepared for the Presidential Task Force on Alternative Formulation of livestock Feed: Product Development, Quality Evaluation and Health Implications. Cabinet Office Lagos, Nigeria.

[33]. Aletor, V.A. (1995). Compositional studies on edible tropical species of Mushrooms. A Review : Food Chemistry. 54:265-26.

[34]. AOAC (2000) Official Method of Analysis (13th edn)). Association of Official Analytical Chemists. Washington, DC USA.

[35]. FAO (1970). Food and Agricultural Organisation (No. 12) FAO, Rome. Italy.

[36]. Griffiths, D.W (1979): The inhibition of digestive enzymes by extracts of field beans (Via fab) J.Sc. Food Agric 30:458 - 462.

[37]. Gropper, S. S., Smith, J. L. and Groff, J. L. (2009) Advanced Nutrition and Human Metabolism. 5th Edition, Australian Wardsworth Cengage Learning.

[38]. Onyeka, E.U. (2013): Food and Nutrition: 3rd ed. Stallmark Media.Owerri.3928.

[39]. Okoye C.O.B (2005). Undergraduate Analytical Chemistry. Dept Pure and Industrial Chemistry UNN, Guidelines and Techniques for analysis of Food. JP Jolyn publishers Nsukka, 98-149

[40]. Yusuf Ali Balami et al (2004) .Practical Manual on Food Technology, Nutrition and Dietetics for Schools and Industries. 2nd Ed. Pub. National Science and Technology Forum, kaduna Polytechnic Pure Culture Techniques, 137-139

[41]. AOAC, (1990): Association of Official Analytical Chemist ( $5^{\text {th }}$ Edn), Washington DC >AOAC.79-104

[42]. Duncan, D. B. (1955).New Multiple range test, Biometrics, 11-42.

[43]. Levesque,R.(2007) SPSS programming and Data management. A guide for SPSS and SAS users ( $4^{\text {th }}$ Edn) Chicago, Illinois: SPSS Inc. ISBN 1-56827-3908Windows versions 2014 software.en.wikipedia. vorg/wiki/spss. Retrieved 31 ${ }^{\text {st }}$ January 2015.

[44]. WHO, (1995). Forty fourth report of WHO expert committee on food additives: Evaluation of certain food additives and contaminants. Geneva, 3234.

[45]. Food and Nutrition Board Institute of Medicine, National Academic (2004) Dietary Reference Intakes (DRI); Recommended Intakes for Individuals. Retrieved 09/06/2009.

[46]. Food and Nutrition Board Institute of Medicine, National Academic (2004) Dietary Reference Intakes (DRI); Recommended Intakes for Individuals. Retrieved 09/06/2009.

[47]. Okoye,COB (2001).Trace Metal Concentrations in Nigerian Fruits and Vegetables .Inter.J.Envron. Studies. vol 58. 501-509.Published by 2001 